\begin{abstract}
HHS Public Access
Author manuscript

J Phys Chem B. Author manuscript; available in PMC 2021 February 06.

Published in final edited form as:

J Phys Chem B. 2020 February 06; 124(5): 840-847. doi:10.1021/acs.jpcb.9b08965.

\section{Comparing Alchemical Free Energy Estimates to Experimental Values Based on the Ben-Naim Formula: How Much Agreement Can We Expect?}

\author{
T. Ryan Rogers, Feng Wang \\ University of Arkansas, Fayetteville, Arkansas;
}

Abstract

The solvation free energy (SFE) plays a key role in thermodynamics. One well-established method for computing the SFE is through an alchemical transformation. However, experimental SFEs are generally determined according to the Ben-Naim equations relying on vapor pressure or density ratios. It is important to establish whether, or to what extent, typical alchemical-based free energy computations provide results comparable to experimental SFEs. In this work, we mimic experimental measurements by simulating the liquid-vapor coexistence of water without alchemical operations. The SFEs measured through vapor pressure and density ratios are used to validate the SFEs obtained through alchemical transformations. It is shown that proper consideration of the nonideal behavior of the vapor is important to ensure that the alchemical SFEs are consistent with the Ben-Naim SFEs. Alchemical transformations in the vapor phase should be performed in addition to solution phase transformations for strongly interacting solutes, such as those with low boiling temperatures and large second virial coefficients. A formula based on the virial expansion of pressure is proposed to provide a better estimate of the true SFE from the simulated vapor pressures. The proposed formula is also applicable to experimental determinations of SFE when the pressure-based route is used.
\end{abstract}

\title{
Graphical Abstract
}

\footnotetext{
Corresponding Author: Feng Wang - University of Arkansas, Fayetteville, Arkansas; fengwang @uark.edu.

Supporting Information

The Supporting Information is available free of charge at https://pubs.acs.org/doi/10.1021/acs.jpcb.9b08965.

Parameters of all fitted equations; number of molecules and box sizes for gas-phase alchemical simulations; derivation of equivalence of chemical potential measured in either constant pressure or constant volume; representative density profile of slab simulations (PDF)

Complete contact information is available at: https://pubs.acs.org/10.1021/acs.jpcb.9b08965

The authors declare no competing financial interest.
} 


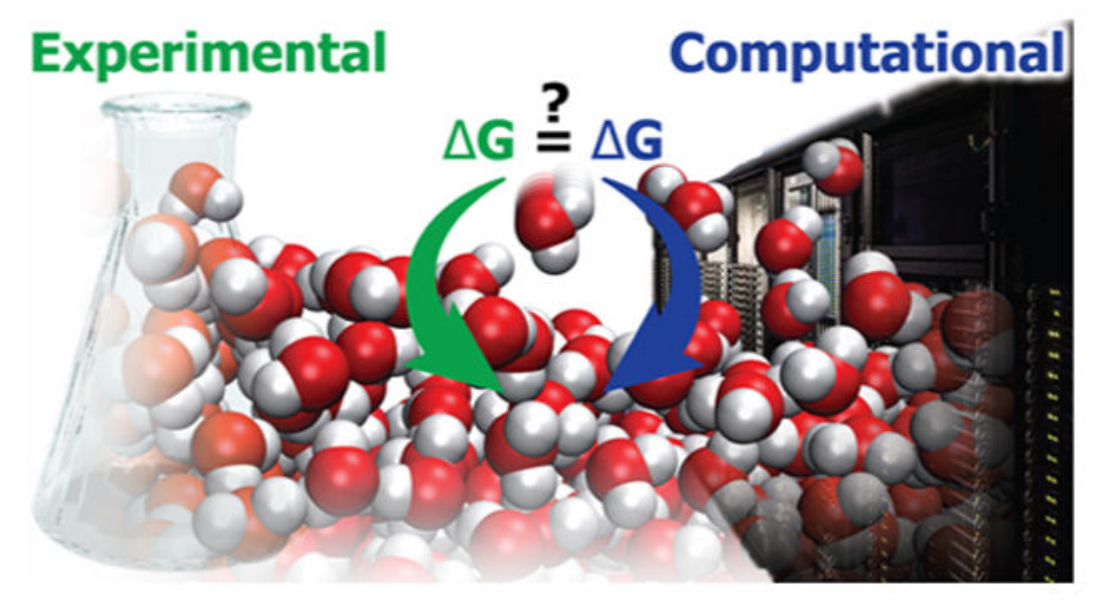

\section{INTRODUCTION}

The solvation free energy (SFE) is one of the most important thermodynamic properties of a molecule. It is affected by the identities and concentrations of the solute and solvent. It directly determines the solubility, vapor pressure, and partition coefficients, which are important in many fields of chemistry. ${ }^{1-4}$ SFE is also important for drug design.., 6 Proteinligand binding is governed by interactions similar to solvation. In addition, the binding of a ligand is associated with desolvation of the ligand as it enters the binding pocket. ${ }^{7}$

It is thus not surprising that the SFE has received tremendous attention in both experimental and theoretical chemistry. Many techniques have been developed for theoretical determination of SFEs, ${ }^{8-13}$ such as Gibbs ensemble Monte Carlo ${ }^{14-16}$ and alchemical growth or annihilation of solutes in the condensed phase. ${ }^{17,18}$ Of the various methods, the alchemical transformation might be the most popular since it is straightforward and most widely implemented in computational packages.

With such an approach, the SFE is measured through either annihilation or creation of a solute in a solvent. The transformation involves the solute fully interacting with the solvent in one state and completely decoupled in the other. Several different methods exist that measure such an alchemical free energy difference, $\Delta G_{\text {alch }}$, such as thermodynamic integration ${ }^{19-22}$ and free energy perturbation. ${ }^{23-25}$

The accuracy of SFE determinations is of paramount importance since SFE appears in the exponents of formulae for many properties, ${ }^{4}$ such as vapor pressure, solubility, and partition coefficients. An error of $R T$ in an SFE would lead to a difference in such properties by a factor of 2.718 .

Since alchemical transformations do not take place in real, benchtop experiments, it is interesting to ask how to properly account for the differences in end states so that an alchemical SFE would agree with an experimental SFE within a small fraction of $R T$, assuming that the force field was perfect. 
For salts and ions in water, the importance of correction factors to the alchemical SFE is well established. ${ }^{26,27}$ Such correction factors ensure that the end points in free energy computations and experimental measurements are fully consistent. For other small molecules, it has also been proposed that certain correction factors should be applied before the theoretical $\Delta G_{\text {alch }}$ is to be reported. ${ }^{28,29}$ A missing correction factor on the order of $R T$ could lead to large percentage errors in SFE for many weakly interacting small molecules. ${ }^{29}$

Experimental SFEs are generally computed based on the relationship proposed by Ben$\mathrm{Naim}^{30}$

$$
\Delta G_{\mathrm{BN}}^{\rho}=\mathrm{RT} \ln \left(\frac{\rho_{\mathrm{g}}}{\rho_{1}}\right)
$$

where $\rho_{\mathrm{l}}$ is the density of the solute in the solution and $\rho_{\mathrm{g}}$ is the gas-phase density of the solute. Equation 1 can be understood using the familiar relationship $\Delta G=-R T \ln (K)$, where $K$ is the equilibrium constant for the solvation process. The SFE is referred to as the interaction work in Ben-Naim's publication. ${ }^{31}$

Since it is generally more convenient to maintain a constant vapor pressure in an experimental setup, ${ }^{32,33}$ the more frequently used formula from the same publication of Ben-Naim is ${ }^{30}$

$$
\Delta G_{\mathrm{BN}}^{P}=R T \ln \left(\frac{P_{\mathrm{vap}}}{R T_{\rho 1}}\right)
$$

where the vapor pressure, $P_{\text {vap }}$, is expected to follow the ideal gas law.

The objective of the present study is to show whether the SFE as measured using eq 1 or 2 indeed fully agrees with that from an alchemical measurement. In other words, are the end points of computational investigations consistent with those assumed experimentally? If the SFEs do not agree, is there a correction factor that could bring them into agreement? Although it is impossible to have a perfect force field so that computed SFEs can be compared to experimental values directly, it is possible to mimic the experimental measurement of SFE using a force field. Such measurements can be performed with bruteforce molecular dynamics (MD) simulations of a liquid-vapor interface. The interface simulations directly measure $\Delta G_{\mathrm{BN}}^{\rho}$ and $\Delta G_{\mathrm{BN}}^{P}$ without the need for particle creation or annihilation.

To the best of our knowledge, there has been no explicit test of the equivalence of the alchemical SFE $\left(\Delta G_{\text {alch }}\right)$ frequently used in theoretical computations and the Ben-Naim SFEs ( $\Delta G_{\mathrm{BN}}^{\rho}$ and $\Delta G_{\mathrm{BN}}^{P}$ ) used by experimentalists. There is also no assessment of whether current SFE calculation procedures, such as those based on the Bennett acceptance ratio (BAR), ${ }^{34,35}$ can converge to the true value without sampling challenges or systematic errors. $2,4,36,37$

In this work, we will thus perform a numerical validation of the equivalence of $\Delta G_{\text {alch }}$ typically used in theoretical work and $\Delta G_{\mathrm{BN}}$ measured by brute-force MD simulations. The 
latter is a proxy for the SFE measured experimentally. For most solutes, converging $\Delta G_{\mathrm{BN}}$ with brute-force MD would be computationally challenging due to the small number of solute molecules, leading to difficulty in determining the precise partitioning between solution and vapor. One system where such a validation can be performed is neat water. The numerical challenge would be further reduced at slightly elevated temperatures where the vapor pressure is higher. Therefore, we will investigate the SFE of water in its own solution in temperatures from $400 \mathrm{~K}$ to near the critical point.

We will use a recently developed water model, BLYSP-4F, ${ }^{38}$ and determine its alchemical SFE, $\Delta G_{\text {alch }}$, using GROMACS ${ }^{39}$ version 2018.4 with the BAR method. To mimic experimental measurement of SFE, a BLYSP-4F water slab is simulated in the temperature range from 400 to $625 \mathrm{~K}$. The "experimental" SFEs of the model water will be determined using the pressure-based route, $\Delta G_{\mathrm{BN}}^{P}$, and the density-based route, $\Delta G_{\mathrm{BN}}^{\rho}$. It is found that proper consideration of nonideality of the gas phase is important for the temperature range studied. Nonideality affects both computational determinations of SFE and experimental SFEs when the more popular pressure-based route is taken.

The remainder of the paper is organized as follows: Section II describes the computational details of the various types of simulations used, and the results of these simulations are discussed in Section III. Section IV concludes with a summary of the key findings from the study.

\section{COMPUTATIONAL DETAILS}

\section{A. Alchemical Free Energy Measurements.}

The alchemical free energies were measured from 400 to $625 \mathrm{~K}$ by switching off Coulombic and van der Waals interactions in said order. The solute-solvent interactions were turned off in 41 equal steps by removing Coulombic interactions in 21 steps followed by van der Waals interactions in 20 steps. For determination of the liquid-phase free energy, the system contained one solute and 749 solvent molecules in a cubic simulation box.

The equations of motion for the alchemical simulations were integrated with a stochastic Langevin integrator ${ }^{40}$ in the NPT ensemble with a 0.5 fs timestep using GROMACS. The pressure at each temperature was set to approximately the equilibrium vapor pressure obtained from slab simulations or $1 \mathrm{bar}$, whichever is higher. The pressure was maintained with the Parrinello-Rahman barostat ${ }^{41,42}$ using a 4 ps relaxation time.

The van der Waals interactions were truncated at $12 \AA$ with long-range corrections for both energy and pressure. Each step in the alchemical simulation was sampled for $5 \mathrm{~ns}$ following a 200 ps equilibration. The BAR method as implemented in GROMACS was used to determine $\Delta G_{\text {alch }}$.

\section{B. Liquid-Vapor Coexistence Simulations.}

In order to mimic experiments, slab configurations of 1300 BLYPSP-4F water molecules were placed in an orthorhombic box of $35 \times 35 \times 100 \AA^{3}$ with liquid-vapor interfaces in the 
$X$ - $Y$ plane. While the thickness of the slab varies with temperature, a $100 \AA$ box leaves about $60 \AA$ of vacuum region at $400 \mathrm{~K}$.

Simulations were performed with GROMACS in the NVT ensemble at 10 temperatures ranging from 400 to $625 \mathrm{~K}$ with $25 \mathrm{~K}$ increments. The equations of motion were integrated with a 0.5 fs timestep. The system temperature was controlled with the Nosé-Hoover thermostat ${ }^{43,44}$ using a 2 ps relaxation time. Each temperature was simulated for at least 100 ns or until the standard error in the gas density became less than $2 \%$ of the average. This amounts to around $440 \mathrm{~ns}$ of simulations at the more challenging temperatures.

Configurations were saved every 50 fs for analysis.

Coulombic interactions were treated using the $3 \mathrm{D}$ particle-mesh Ewald method ${ }^{45}$ with a sixth-order spline and a real space precision of $10^{-6}$; the Ewald reciprocal space contribution was integrated with a Fourier mesh density of $0.12 \mathrm{~nm}^{-1}$. The version of GROMACS does not support Ewald treatment for van der Waals interactions when tabulated intermolecular potentials are used as with our model. The standard long-range van der Waals correction is not applicable to inhomogeneous systems and thus is not used for our slab simulations. A long cutoff of $17.5 \AA$ was used instead, following the recommendation of $\mathrm{Hu}$ and Wang, 46 which leads to surface tension to be converged within $2 \mathrm{mN} / \mathrm{m}$ for the water model.

\section{Measurement of Densities and Vapor Pressures.}

Liquid and gas densities were obtained from the slab simulations, following the protocol developed by $\mathrm{Hu}$ and Wang. ${ }^{46}$ Briefly, the liquid phase is identified with a $30 \AA$ thick window with the greatest average density. To avoid interface contributions, the liquid density is measured as the average density of a $10 \AA$ wide region in the center of the $30 \AA$ window. The gas density is the average density of a $10 \AA$ region half a box length away from the center of the liquid. Care was taken to remove center of mass drift of the slabs. A representative density profile for the slab at $425 \mathrm{~K}$ is shown in the Supporting Information.

The difference of liquid and gas densities was fitted to a sixth-order truncation of the Wegner scaling law 47,48

$$
\rho_{1}-\rho_{\mathrm{g}}=\sum_{k=0}^{5} A_{k}|\tau|^{\beta+k \Delta}
$$

where $\tau$ is defined as

$$
\tau=1-\frac{T}{T_{\mathrm{C}}}
$$

where $T_{\mathrm{C}}$ is the critical temperature, which is treated as a fitting parameter along with the coefficients $A_{k}$. While fitting eq $3, \beta$ and $\Delta$ were chosen to be 0.325 and 0.5 , respectively, according to the $3 \mathrm{D}$ Ising universality class. ${ }^{49,50}$

Similarly, the sum of densities was fitted to a sixth-order expansion of the form ${ }^{47,48}$ 


$$
\rho_{1}+\rho_{\mathrm{g}}=2 \rho_{\mathrm{C}}+D_{1-\alpha}|\tau|^{1-\alpha}+D_{0}|\tau|+\sum_{k=1}^{3} D_{k}|\tau|^{1-\alpha+k \Delta}
$$

in which $a$ was chosen to be 0.11 following Ley-Koo and Green. ${ }^{48}$ In addition to the critical density, $\rho_{\mathrm{C}}, D_{1-a}, D_{0}$, and $D_{k}$ are also fitting parameters. $T_{\mathrm{C}}$ was obtained by fitting eq 3 . The measured densities for the gas and liquid are reported in Table 1 with the fitted densities compared to the measured values in Figure 1.

Saturation vapor pressures were obtained from the slab simulations by measuring the component of the stress tensor normal to the slab surface. The simulated pressures are also reported in Table 1. While vapor pressures are frequently fitted to Antoine's equation ${ }^{51}$ in a limited temperature range, Wagner's equation ${ }^{52}$ is able to fit the entire temperature range studied in this work. Wagner's empirical correlation has the form

$$
\ln \left(P_{\text {vap }}\right)=\ln \left(P_{\mathrm{C}}\right)+\frac{T_{\mathrm{C}}}{T}\left(\xi_{1}|\tau|+\xi_{1.5}|\tau|^{1.5}+\xi_{3}|\tau|^{3}+\xi_{6}|\tau|^{6}\right)
$$

The fitting parameters of eq 6 are $P_{\mathrm{C}}$ (the critical pressure) and $\xi_{i}$. The quality of the fit is shown in Figure 2.

With this fitting procedure, the critical point was determined to be $674.6 \mathrm{~K}$ at 146.9 bar and compares favorably to that reported previously of $685.1 \pm 3.9 \mathrm{~K}$ and $189.8 \pm 48.7$ bar for the BLYPSP-4F model. ${ }^{46}$ The fitted parameters for eqs 3-6 are reported in the Supporting Information.

\section{Calculation of Virial Coefficients.}

As will be discussed later, the virial equation of state will be used to account for the deviations from ideal gas behavior of BLYPSP-4F water's pressure and density correlations. For this purpose, the second and third virial coefficients, $B_{2}$ and $B_{3}$, of BLYPSP-4F water were calculated using Mayer Sampling Monte Carlo (MSMC) $)^{53}$ as implemented in a customized version of the Etomica ${ }^{54}$ software package. A minimum of $10^{8}$ MSMC steps was used to estimate the virial coefficients at each temperature over the same range as the slab simulations. To reduce noise in the estimates, the temperature dependence of the second virial coefficient was fitted to Eubank's correlation function ${ }^{55}$

$$
B_{2}(T)=\psi_{0}-\frac{\psi_{1}}{T} \exp \left(\frac{\psi_{2}}{T^{2}+\psi_{3}}\right)
$$

where $\psi_{i}$ are the fitting parameters. The MSMC virial coefficients are given in Table 2, and the fitted parameters of eq 7 are reported in the Supporting Information. The agreement between measured and fitted $B_{2}$ is shown in Figure 3. While Harvey and Lemmon56 have used a different correlation function for fitting the second virial coefficient, Eubank's equation fits our data better than Harvey's equation with up to four parameters. 


\section{RESULTS AND DISCUSSION}

In order to determine if the alchemical free energy is comparable to the experimental SFE based on the Ben-Naim definitions, $\Delta G_{\mathrm{BN}}^{P}$ and $\Delta G_{\mathrm{BN}}^{\rho}$ were calculated with simulated data with the abovementioned fittings. The use of fitted data helps to reduce statistical noise due to fluctuations. The comparison of $\Delta G_{\mathrm{alch}}, \Delta G_{\mathrm{BN}}^{P}$, and $\Delta G_{\mathrm{BN}}^{\rho}$ is presented in Figure 4.

$\Delta G_{\text {alch }}$ is best compared to $\Delta G_{\mathrm{BN}}^{\rho}$ since no assumption regarding the gas equation of state is made for the determination of $\Delta G_{\mathrm{BN}}^{\rho}$. As it is clear from Figure 4, the two free energy estimates disagree except at $400 \mathrm{~K}$, with the difference increasing as temperature increases. It would be difficult to judge at this stage if the agreement at $400 \mathrm{~K}$ is merely coincidental.

To understand the observed difference in SFEs, we consider implications of the alchemical simulations. The SFE is defined as the free energy change for the process

$$
\text { solute }(\mathrm{g}) \rightarrow \text { solute(solution) }
$$

A typical alchemical SFE measurement only performs the alchemical simulation in the solution phase. The reference state is a noninteracting solute, which is equivalent to an ideal gas state. In reality, a gas-phase molecule does interact weakly. The larger deviation of $\Delta G_{\text {alch }}$ from $\Delta G_{\mathrm{BN}}^{\rho}$ at higher temperature could be a result of interactions between gas molecules as the vapor density increases. ${ }^{57,58}$ To test this hypothesis, the free energy for an alchemical transformation in the vapor phase was also measured with the BAR method.

It is worth noting that gas-phase alchemical free energy estimates have their own numerical challenges. For example, the variance in volume in an NPT simulation is proportional to the isothermal compressibility, which is significantly larger for a gas than for a liquid. Many barostats would have trouble with such large volume fluctuations, and therefore, gas-phase alchemical measurements were performed in the NVT ensemble. The free energy change of such a process is the Helmholtz free energy. However, assuming a sufficiently large number of molecules and an equation of state not deviating significantly from the corresponding ideal gas behavior, it can be shown that the Gibbs free energy change in a hypothetical constant pressure simulation should be the same as the change in Helmholtz free energy measured in the constant volume ensemble. In the Supporting Information, it is shown that, for all practical purposes, the difference between the Helmholtz free energy computed and the desired Gibbs free energy can be ignored. We therefore refer to alchemical free energies computed for interacting gas systems as $\Delta G_{\text {alch }}^{\mathrm{g}}$ and distinguish these from the aqueous-phase free energies, $\Delta G_{\text {alch }}^{1}$.

The gas-phase $\Delta G_{\text {alch }}^{\mathrm{g}}$ is measured by a protocol similar to that used for the liquid-phase $\Delta G_{\text {alch. }}^{1}$ At temperatures above $550 \mathrm{~K}$, the number of water molecules in the box is chosen so that the gas-phase simulation uses a box of similar volume to the liquid phase. At $550 \mathrm{~K}$ and below, 10 water molecules are used to solvate the water molecule being transformed, with 
the box size chosen to reproduce the vapor density according to eqs $3-5$. The number of molecules in the box and the box volume used are summarized in Table S2 of the Supporting Information. The boxes with fewer gas-phase molecules require longer simulation times to converge $\Delta G_{\text {alch }}^{\mathrm{g}}$. Each alchemical step was simulated from 15 to $30 \mathrm{~ns}$ depending on the temperature, after a $1 \mathrm{~ns}$ equilibration period. With the gas-phase contribution, the alchemical estimate of the hydration free energy becomes

$$
\Delta G_{\mathrm{alch}}^{\prime}=\Delta G_{\mathrm{alch}}^{\mathrm{l}}-\Delta G_{\mathrm{alch}}^{\mathrm{g}}
$$

where the prime sign denotes that the gas-phase contributions were computed explicitly. $\Delta G_{\text {alch }}^{\prime}$ is compared to $\Delta G_{\mathrm{BN}}^{\rho}$ in Figure 5. Clearly, $\Delta G_{\text {alch }}^{\prime}$ agrees with the Ben-Naim $\Delta G_{\mathrm{BN}}^{\rho}$ within a negligible fraction of the thermal energy at $400 \mathrm{~K}$.

While excellent agreement is achieved between $\Delta G_{\mathrm{alch}}^{\prime}$ and $\Delta G_{\mathrm{BN}}^{\rho}$, it is also important to reconcile the difference between $\Delta G_{\mathrm{BN}}^{P}$ and $\Delta G_{\text {alch }}^{\prime}$ since most experimental estimates of SFE are based on $\Delta G_{\mathrm{BN}}^{P}$. Although the computation of $\Delta G_{\mathrm{BN}}^{P}$ did not assume noninteracting gasphase molecules, it still assumes the ideal gas equation of state, $P_{\text {vap }}=\rho_{\mathrm{g}} R T$. A better approximation will be to use the virial equation of state

$$
P_{\mathrm{vap}}=\rho_{\mathrm{g}} R T\left(1+B_{2} \rho_{\mathrm{g}}+B_{3} \rho_{\mathrm{g}}^{2}+\cdots\right)
$$

where $B_{2}$ and $B_{3}$ are the second and third virial coefficients, respectively, and higher-order terms are omitted.

Along with $B_{2}$ and $B_{3}$, Table 2 also reports the second and third terms in eq 10 calculated based on the saturation vapor density for each temperature. Clearly, the third-order contribution is much smaller than the second-order contribution. Although an algebraic form for the gas density can be derived based on the third-order virial truncation, for all practical purposes, truncation at the second-order should be sufficient.

Truncating eq 10 to the second-order term, it can be shown that

$$
\rho_{\mathrm{g}}=-\sqrt{\frac{P_{\mathrm{vap}}}{R T B_{2}}+\left(\frac{1}{2 B_{2}}\right)^{2}}-\frac{1}{2 B_{2}}
$$

where $P_{\text {vap }}$ is the saturation vapor pressure used to calculate $\Delta G_{\mathrm{BN}}^{P}$. The revised equation for the SFE is thus

$$
\Delta G^{\prime}{ }_{\mathrm{BN}}^{P}=R T \ln \left[\left(-\sqrt{\frac{P_{\mathrm{vap}}}{R T B_{2}}+\left(\frac{1}{2 B_{2}}\right)^{2}}-\frac{1}{2 B_{2}}\right) / \rho_{1}\right]
$$

Figure 6 compares $\Delta G^{\prime}{ }_{\mathrm{BN}}^{P}$ and $\Delta G_{\mathrm{BN}}^{\rho}$. Much better agreement is achieved, especially at higher temperatures, once the virial equation of state is used to approximate the gas density. At $400 \mathrm{~K}$, the measured vapor pressure is 0.035 bar with a vapor density of $0.0161 \mathrm{~mol} / \mathrm{L}$. In 
the vacuum region of our simulation, there are on average 0.7 waters present. Thus, the statistical noise on both the vapor density and pressure measurements is very large. Uncertainty is expected also on our estimate of the error bar. Since $\Delta G_{\text {alch }}^{\prime}$ is in good agreement with $\Delta G_{\mathrm{BN}}^{\rho}$, we anticipate that the density measurement is properly converged. The measurement of $P_{\text {vap }}$ is likely not, which explains the small deviation at lower temperatures in Figure 6. The standard deviation of the normal pressure in our simulations is more than $400 \mathrm{bar}$ at $400 \mathrm{~K}$. Measuring normal pressure in the presence of the coexisting liquid with an accuracy much smaller than 0.035 bar is thus expected to be challenging. Given the challenge of converging vapor pressures in the presence of a liquid phase, we anticipate an equilibrium pressure estimate based on alchemical SFE using eq 2 would be numerically more reliable at lower temperatures.

We note that experimental SFEs are generally calculated based on eq 2 assuming the ideal gas equation of state. For liquid water, the SFE in its own solution is the same as the chemical potential, $\mu$. In the Ben-Naim work (refs 30,31 ), the calculation of $\mu$ of water assumed the ideal gas equation of state. Table 3 compares the experimental $\mu$ of water calculated based on eqs 1,2 , and $12 . \Delta G_{\mathrm{BN}}^{P}$ is $\mu$ of water typically reported in standard reference books. $\Delta G_{\mathrm{BN}}^{\rho}$ is computed using the experimental density of real water, which is directly comparable to the alchemical computations when the correction to the gas-phase interactions is properly considered. $\Delta G^{\prime}{ }_{\mathrm{BN}}^{P}$ is computed via eq 12 using experimental vapor pressures and second virial coefficients. Fortunately, deviation from the ideal gas equation of state is not very large for real water, leading to an error of only $0.014 \%$ between $\Delta G_{\mathrm{BN}}^{P}$ and $\Delta G_{\mathrm{BN}}^{\rho}$ at $25^{\circ} \mathrm{C}$. This error increases to $0.2 \%$ at $100{ }^{\circ} \mathrm{C}$ and $2 \%$ at $200{ }^{\circ} \mathrm{C}$. The use of eq 12 gives very good agreement to eq 1 based on experimental density of neat water. We note that, for molecules with strong gas-phase interactions, the error introduced by using $\Delta G_{\mathrm{BN}}^{P}$, as in many experimental studies, could be large. For example, for 1,3-butadiene, our calculations based on experimental virial coefficients indicate an error up to $14 \%$ at $25{ }^{\circ} \mathrm{C}$ if eq 2 is used to estimate the experimental SFE instead of eq 12.

\section{SUMMARY AND CONCLUSIONS}

In this study, the SFE of water in its own solution was computed both by alchemical transformations and by applying to simulated data the same formulae used to define experimental SFEs. Typical alchemical free energy measurements only perform alchemical transformations in the liquid phase. For BLYPSP-4F water, this was found to be inadequate at elevated temperatures above $425 \mathrm{~K}$. Alchemical transformations in the vapor phase must be performed to achieve good agreement with experimental SFEs if solute-solute interactions are strong, which occurs when the solute density is high. We anticipate that solutes with lower boiling points likely warrant vapor-phase alchemical transformations for accurate free energy determinations.

We note that there are two types of nonideality corrections. The alchemical free energy measurements correct for gas-phase interactions, while the second viral-based formula 
corrects for deviation from the ideal gas equation of state. Although gas-phase interactions lead to deviation from the ideal gas equation of state, the two types of corrections are related but not the same. Typical experimental SFEs based on vapor-phase pressure measurements, $\Delta G_{\mathrm{BN}}^{P}$, assume the ideal gas equation of state. A better estimate of the experimental SFE can be obtained through eq 12 instead of eq 2 , using the more accurate virial equation of state.

The study also serves as a confidence check confirming that, with care, the alchemical SFE calculated with standard alchemical procedures should be able to provide excellent agreement with experimental values when nonideal gas behavior is either small or properly accounted for. For small molecules, if a model fails to give good agreement with experimental references, the cause is more likely to be the quality of the force field than uncertainty in the alchemical free energy determination. ${ }^{60}$

\section{Supplementary Material}

Refer to Web version on PubMed Central for supplementary material.

\section{ACKNOWLEDGMENTS}

This work was supported by the National Institutes of Health under grant no. 1R01GM120578. Computational resources were provided by the Arkansas High Performance Computing Center through grant MRI-R2 \#09559124 provided by the NSF; partial funding for the computational resource is provided by the Arkansas Bioscience Institute. This material is based upon work supported by the National Science Foundation Graduate Research Fellowship Program under Grant No. 1647782. T.R.R. would like to thank Jicun Li for helpful discussions about alchemical simulations. The authors also thank David Kofke and Andrew Schultz for help in obtaining the Etomica code for the virial coefficient calculations.

\section{REFERENCES}

(1). Beveridge DL; Dicapua FM Free Energy Via Molecular Simulation: Applications to Chemical and Biomolecular Systems. Annu. Rev. Biophys. Biophys. Chem 1989, 18, 431-492. [PubMed: 2660832]

(2). Duarte Ramos Matos G; Kyu DY; Loeffler HH; Chodera JD; Shirts MR; Mobley DL Approaches for Calculating Solvation Free Energies and Enthalpies Demonstrated with an Update of the Freesolv Database. J. Chem. Eng. Data 2017, 62, 1559-1569. [PubMed: 29056756]

(3). Ratkova EL; Fedorov MV Combination of Rism and Cheminformatics for Efficient Predictions of Hydration Free Energy of Polyfragment Molecules: Application to a Set of Organic Pollutants. J. Chem. Theory Comput 2011, 7, 1450-1457. [PubMed: 26610135]

(4). Ratkova EL; Palmer DS; Fedorov MV Solvation Thermodynamics of Organic Molecules by the Molecular Integral Equation Theory: Approaching Chemical Accuracy. Chem. Rev 2015, 115, 6312-6356. [PubMed: 26073187]

(5). Yin J; Henriksen NM; Slochower DR; Shirts MR; Chiu MW; Mobley DL; Gilson MK Overview of the Sampl5 Host-Guest Challenge: Are We Doing Better? J. Comput.-Aided Mol. Des 2017, 31, 1-19. [PubMed: 27658802]

(6). Wang L; Wu Y; Deng Y; Kim B; Pierce L; Krilov G; Lupyan D; Robinson S; Dahlgren MK; Greenwood J; et al. Accurate and Reliable Prediction of Relative Ligand Binding Potency in Prospective Drug Discovery by Way of a Modern Free-Energy Calculation Protocol and Force Field. J. Am. Chem. Soc 2015, 137, 2695-2703. [PubMed: 25625324]

(7). Hamelberg D; McCammon JA Standard Free Energy of Releasing a Localized Water Molecule from the Binding Pockets of Proteins: Double-Decoupling Method. J. Am. Chem. Soc 2004, 126, 7683-7689. [PubMed: 15198616] 
(8). Rowley RL; Shupe TD; Schuck MW A Direct Method for Determination of Chemical Potential from Osmotic Molecular Dynamics Simulations. Fluid Phase Equilib 1995, 104, 159-171.

(9). Varilly P; Chandler D Water Evaporation: A Transition Path Sampling Study. J. Phys. Chem. B 2013, 117, 1419-1428. [PubMed: 23294322]

(10). Wilson MA; Pohorille A Adsorption and Solvation of Ethanol at the Water Liquid-Vapor Interface: A Molecular Dynamics Study. J. Phys. Chem. B 1997, 101, 3130-3135. [PubMed: 11540504]

(11). Song J; Qiu L; Zhang JZH An Efficient Method for Computing Excess Free Energy of Liquid. Sci. China: Chem 2018, 61, 135-140.

(12). Do H; Hirst JD; Wheatley RJ Rapid Calculation of Partition Functions and Free Energies of Fluids. J. Chem. Phys 2011, 135, 174105. [PubMed: 22070290]

(13). Christ CD; van Gunsteren WF Simple, Efficient, and Reliable Computation of Multiple Free Energy Differences from a Single Simulation: A Reference Hamiltonian Parameter Update Scheme for Enveloping Distribution Sampling (Eds). J. Chem. Theory Comput 2009, 5, 276-286. [PubMed: 26610104]

(14). Martin MG; Siepmann JI Calculating Gibbs Free Energies of Transfer from Gibbs Ensemble Monte Carlo Simulations. Theor. Chem. Acc 1998, 99, 347-350.

(15). Shi W; Maginn EJ Improvement in Molecule Exchange Efficiency in Gibbs Ensemble Monte Carlo: Development and Implementation of the Continuous Fractional Component Move. J. Comput. Chem 2008, 29, 2520-2530. [PubMed: 18478586]

(16). Panagiotopoulos AZ Direct Determination of Phase Coexistence Properties of Fluids by Monte Carlo Simulation in a New Ensemble. Mol. Phys 1987, 61, 813-826.

(17). Straatsma TP; McCammon JA Computational Alchemy. Annu. Rev. Phys. Chem 1992, 43, 407435.

(18). Meng Y; Sabri Dashti D; Roitberg AE Computing Alchemical Free Energy Differences with Hamiltonian Replica Exchange Molecular Dynamics (H-Remd) Simulations. J. Chem. Theory Comput 2011, 7, 2721-2727. [PubMed: 22125475]

(19). Lawrenz M; Baron R; McCammon JA Independent-Trajectories Thermodynamic-Integration Free-Energy Changes for Biomolecular Systems: Determinants of H5n1 Avian Influenza Virus Neuraminidase Inhibition by Peramivir. J. Chem. Theory Comput 2009, 5, 1106-1116. [PubMed: 19461872]

(20). Bhati AP; Wan S; Wright DW; Coveney PV Rapid, Accurate, Precise, and Reliable Relative Free Energy Prediction Using Ensemble Based Thermodynamic Integration. J. Chem. Theory Comput 2017, 13, 210-222. [PubMed: 27997169]

(21). Hahn DF; Hünenberger PH Alchemical Free-Energy Calculations by Multiple-Replica $\lambda$ Dynamics: The Conveyor Belt Thermodynamic Integration Scheme. J. Chem. Theory Comput 2019, 15, 2392-2419. [PubMed: 30821973]

(22). Heidari M; Kremer K; Cortes-Huerto R; Potestio R Spatially Resolved Thermodynamic Integration: An Efficient Method to Compute Chemical Potentials of Dense Fluids. J. Chem. Theory Comput 2018, 14, 3409-3417. [PubMed: 29874069]

(23). Shirts MR; Pande VS Comparison of Efficiency and Bias of Free Energies Computed by Exponential Averaging, the Bennett Acceptance Ratio, and Thermodynamic Integration. J. Chem. Phys 2005, 122, 144107. [PubMed: 15847516]

(24). Kuwajima S; Kikuchi H; Fukuda M Molecular-Dynamics Evaluation of Fluid-Phase Equilibrium Properties by a Novel Free-Energy Perturbation Approach: Application to Gas Solubility and Vapor Pressure of Liquid Hexane. J. Chem. Phys 2006, 124, 124111. [PubMed: 16599666]

(25). Jarzynski C Nonequilibrium Equality for Free Energy Differences. Phys. Rev. Lett 1997, 78, 2690-2693.

(26). Li J; Wang F Accurate Prediction of the Hydration Free Energies of 20 Salts through Adaptive Force Matching and the Proper Comparison with Experimental References. J. Phys. Chem. B 2017, 121, 6637-6645. [PubMed: 28621540]

(27). Benavides AL; Portillo MA; Chamorro VC; Espinosa JR; Abascal JLF; Vega C A Potential Model for Sodium Chloride Solutions Based on the Tip4p/2005 Water Model. J. Chem. Phys 2017, 147, 104501. [PubMed: 28915761] 
(28). Horn HW; Swope WC; Pitera JW Characterization of the Tip4p-Ew Water Model: Vapor Pressure and Boiling Point. J. Chem. Phys 2005, 123, 194504. [PubMed: 16321097]

(29). Vega C; Noya EG Revisiting the Frenkel-Ladd Method to Compute the Free Energy of Solids: The Einstein Molecule Approach. J. Chem. Phys 2007, 127, 154113. [PubMed: 17949138]

(30). Ben-Naim A; Marcus Y Solvation Thermodynamics of Nonionic Solutes. J. Chem. Phys 1984, 81, 2016-2027.

(31). Ben-Naim AY Solvation Thermodynamics; Plenum Press: New York, 1987.

(32). McAuliffe C Solubility in Water of Paraffin, Cycloparaffin, Olefin, Acetylene, Cycloolefin, and Aromatic Hydrocarbons. J. Phys. Chem 1966, 70, 1267-1275.

(33). Robbins GA; Wang S; Stuart JD Using the Static Headspace Method to Determine Henry's Law Constants. Anal. Chem 1993, 65, 3113-3118.

(34). Bennett CH Efficient Estimation of Free Energy Differences from Monte Carlo Data. J. Comput. Phys 1976, 22, 245-268.

(35). Shirts MR; Chodera JD Statistically Optimal Analysis of Samples from Multiple Equilibrium States. J. Chem. Phys 2008, 129, 124105. [PubMed: 19045004]

(36). Zuckerman DM; Woolf TB Theory of a Systematic Computational Error in Free Energy Differences. Phys. Rev. Lett 2002, 89, 180602. [PubMed: 12398588]

(37). Mobley DL; Klimovich PV Perspective: Alchemical Free Energy Calculations for Drug Discovery. J. Chem. Phys 2012, 137, 230901. [PubMed: 23267463]

(38). Wang F; Akin-Ojo O; Pinnick E; Song Y Approaching Post-Hartree-Fock Quality Potential Energy Surfaces with Simple Pair-Wise Expressions: Parameterising Point-Charge-Based Force Fields for Liquid Water Using the Adaptive Force Matching Method. Mol. Simul 2011, 37, 591605.

(39). Van der Spoel D; Lindahl E; Hess B; Groenhof G; Mark AE; Berendsen HJC Gromacs: Fast, Flexible, and Free. J. Comput. Chem 2005, 26, 1701-1718. [PubMed: 16211538]

(40). Goga N; Rzepiela AJ; de Vries AH; Marrink SJ; Berendsen HJC. Efficient Algorithms for Langevin and Dpd Dynamics. J. Chem. Theory Comput 2012, 8, 3637-3649. [PubMed: 26593009]

(41). Parrinello M; Rahman A Polymorphic Transitions in Single Crystals: A New Molecular Dynamics Method. J. Appl. Phys 1981, 52, 7182-7190.

(42). Nosé S; Klein ML Constant Pressure Molecular Dynamics for Molecular Systems. Mol. Phys 1983, 50, 1055-1076.

(43). Nosé S A Molecular-Dynamics Method for Simulations in the Canonical Ensemble. Mol. Phys $1984,52,255-268$.

(44). Hoover WG Canonical Dynamics: Equilibrium Phase-Space Distributions. Phys. Rev. A 1985, 31, 1695-1697.

(45). Darden T; York D; Pedersen L Particle Mesh Ewald - an N. Log(N) Method for Ewald Sums in Large Systems. J. Chem. Phys 1993, 98, 10089-10092.

(46). Hu H; Wang F The Liquid-Vapor Equilibria of Tip4p/2005 and Blypsp-4f Water Models Determined through Direct Simulations of the Liquid-Vapor Interface. J. Chem. Phys 2015, 142, 214507. [PubMed: 26049508]

(47). Wegner FJ Corrections to Scaling Laws. Phys. Rev. B 1972, 5, 4529.

(48). Ley-Koo M; Green MS Consequences of the Renormalization Group for the Thermodynamics of Fluids near the Critical Point. Phys. Rev. A 1981, 23, 2650-2659.

(49). Guggenheim EA The Principle of Corresponding States. J. Chem. Phys 1945, 13, 253-261.

(50). Guissani Y; Guillot B A Computer-Simulation Study of the Liquid-Vapor Coexistence Curve of Water. J. Chem. Phys 1993, 98, 8221-8235.

(51). Antoine C Vapor Pressure: A New Relationship between Pressure and Temperature. CR Acad. Sci 1888, 107, 681-685.

(52). Wagner W New Vapor Pressure Measurements for Argon and Nitrogen and a New Method for Establishing Rational Vapor Pressure Equations. Cryogenics 1973, 13, 470-482.

(53). Singh JK; Kofke DA Mayer Sampling: Calculation of Cluster Integrals Using Free-Energy Perturbation Methods. Phys. Rev. Lett 2004, 92, 220601. [PubMed: 15245206] 
(54). Schultz AJ; Kofke DA Etomica: An Object-Oriented Framework for Molecular Simulation. J. Comput. Chem 2015, 36, 573-583. [PubMed: 25565378]

(55). Eubank PT; Joffrion LL; Patel MR; Warowny W Experimental Densities and Virial Coefficients for Steam from 348 to $498 \mathrm{~K}$ with Correction for Adsorption Effects. J. Chem. Thermodyn 1988, 20, 1009-1034.

(56). Harvey AH; Lemmon EW Correlation for the Second Virial Coefficient of Water. J. Phys. Chem. Ref. Data 2004, 33, 369-376.

(57). Wierzchowski SJ; Kofke DA Fugacity Coefficients of Saturated Water from Molecular Simulation. J. Phys. Chem. B 2003, 107, 12808-12813.

(58). Fuerst GB; Ley RT; Paluch AS Calculating the Fugacity of Pure, Low Volatile Liquids Via Molecular Simulation with Application to Acetanilide, Acetaminophen, and Phenacetin. Ind. Eng. Chem. Res 2015, 54, 9027-9037.

(59). Wagner W; Pruss A The Iapws Formulation 1995 for the Thermodynamic Properties of Ordinary Water Substance for General and Scientific Use. J. Phys. Chem. Ref. Data 2002, 31, 387-535.

(60). Mobley DL Let's Get Honest About Sampling. J. Comput.- Aided Mol. Des 2012, 26, 93-95. [PubMed: 22113833] 


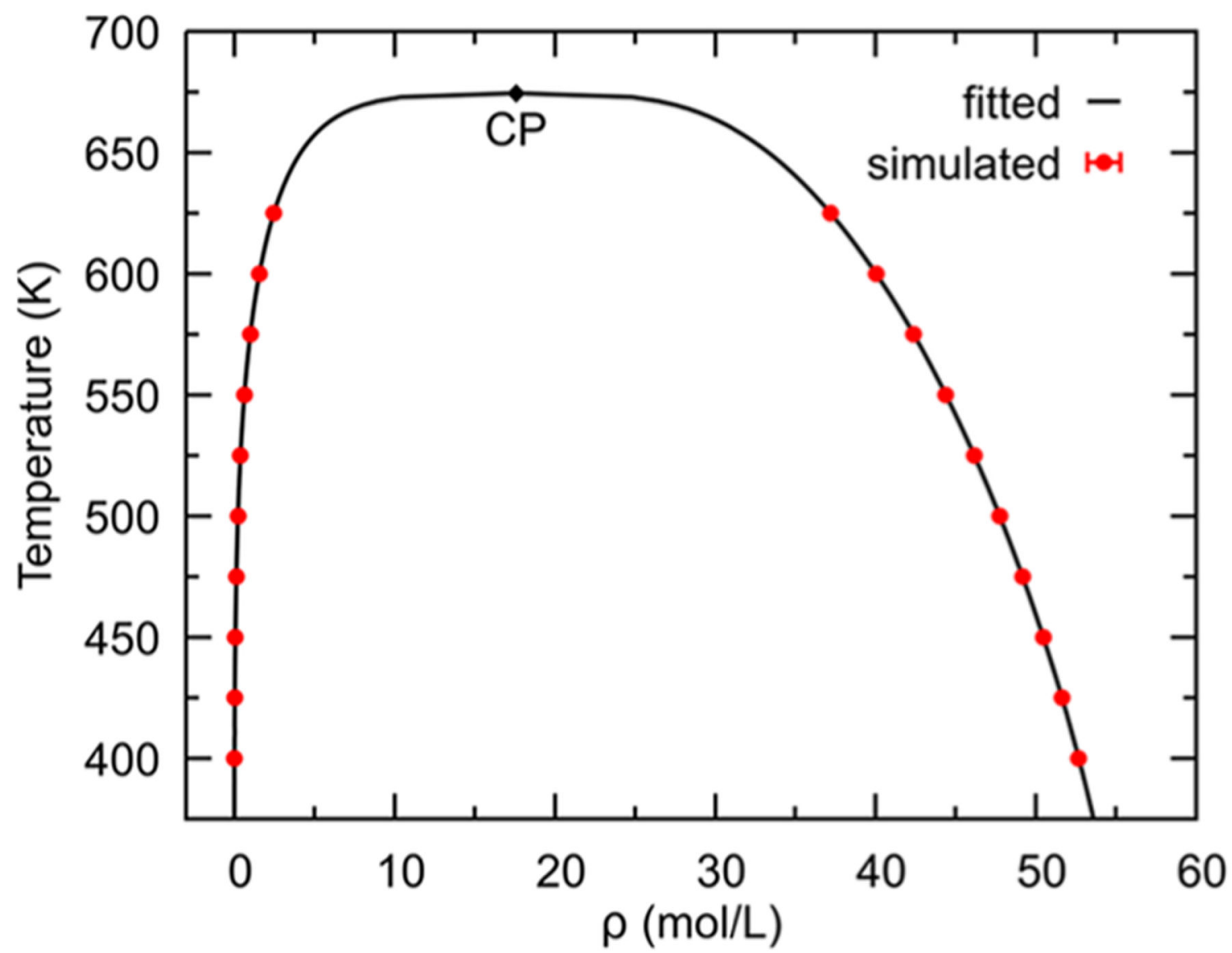

Figure 1.

Phase diagram of BLYPSP-4F water, comparing densities measured from liquid-vapor coexistence simulations (red circles) and the values predicted from the fitted Wegner scaling law (black curve). The fitted critical point, at $17.57 \mathrm{~mol} / \mathrm{L}$ and $674.6 \mathrm{~K}$, is labeled "CP." All error bars are smaller than the size of the data symbols. 


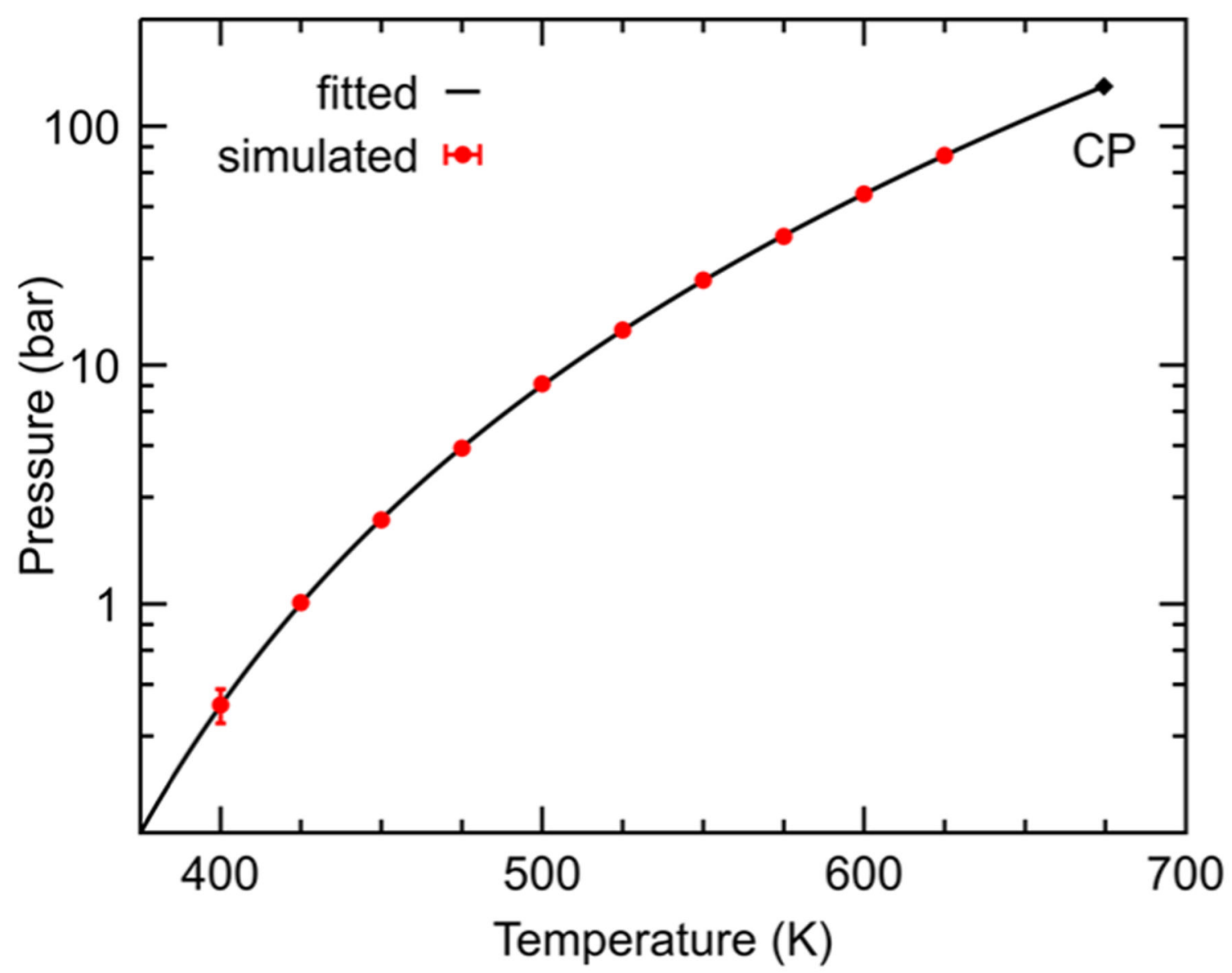

Figure 2.

Simulated vapor pressures fitted to Wagner's empirical correlation function. The critical point at $674.6 \mathrm{~K}$ and 146.9 bar, determined by the fitting procedures outlined in the text, is marked with "CP." 


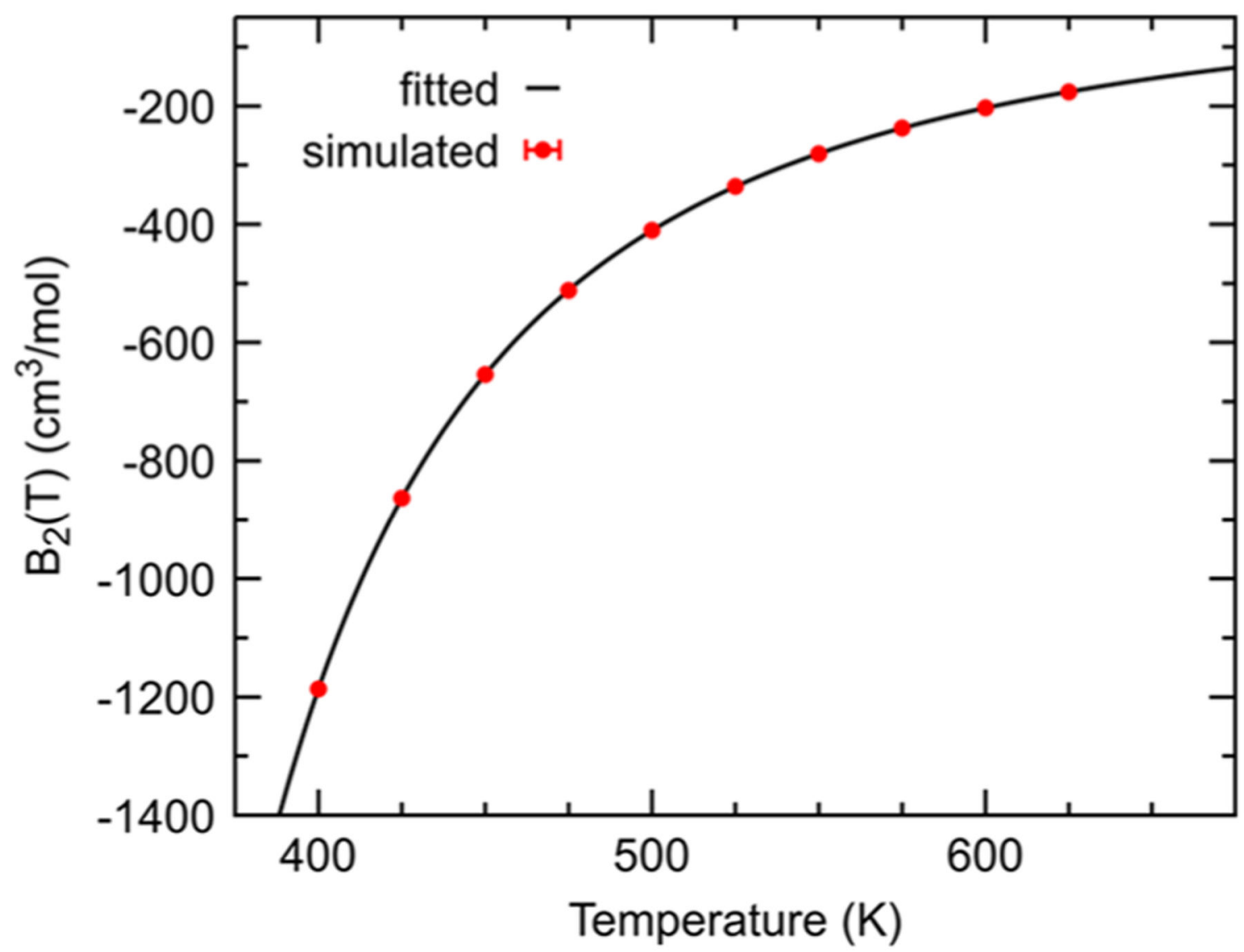

Figure 3.

Temperature-dependent second virial coefficients of BLYPSP-4F water (red points) fitted to Eubank's correlation function (black curve). All error bars are smaller than the simulated data symbols. 


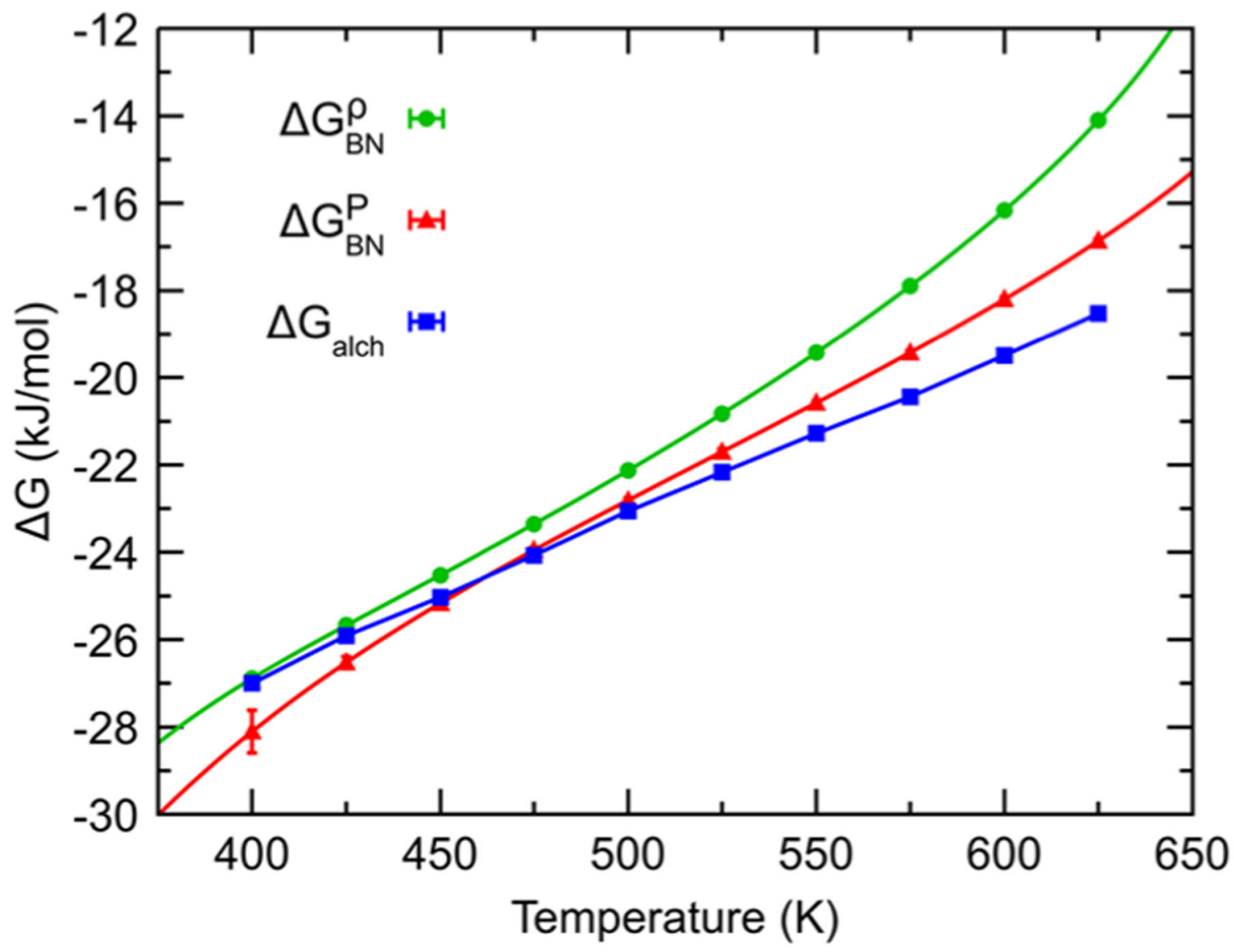

Figure 4.

Solvation free energies of water determined through alchemical simulations, $\Delta G_{\mathrm{alch}}$ (blue), compared with the Ben-Naim estimates, $\Delta G_{\mathrm{BN}}^{\rho}$ (green) and $\Delta G_{\mathrm{BN}}^{P}$ (red). 


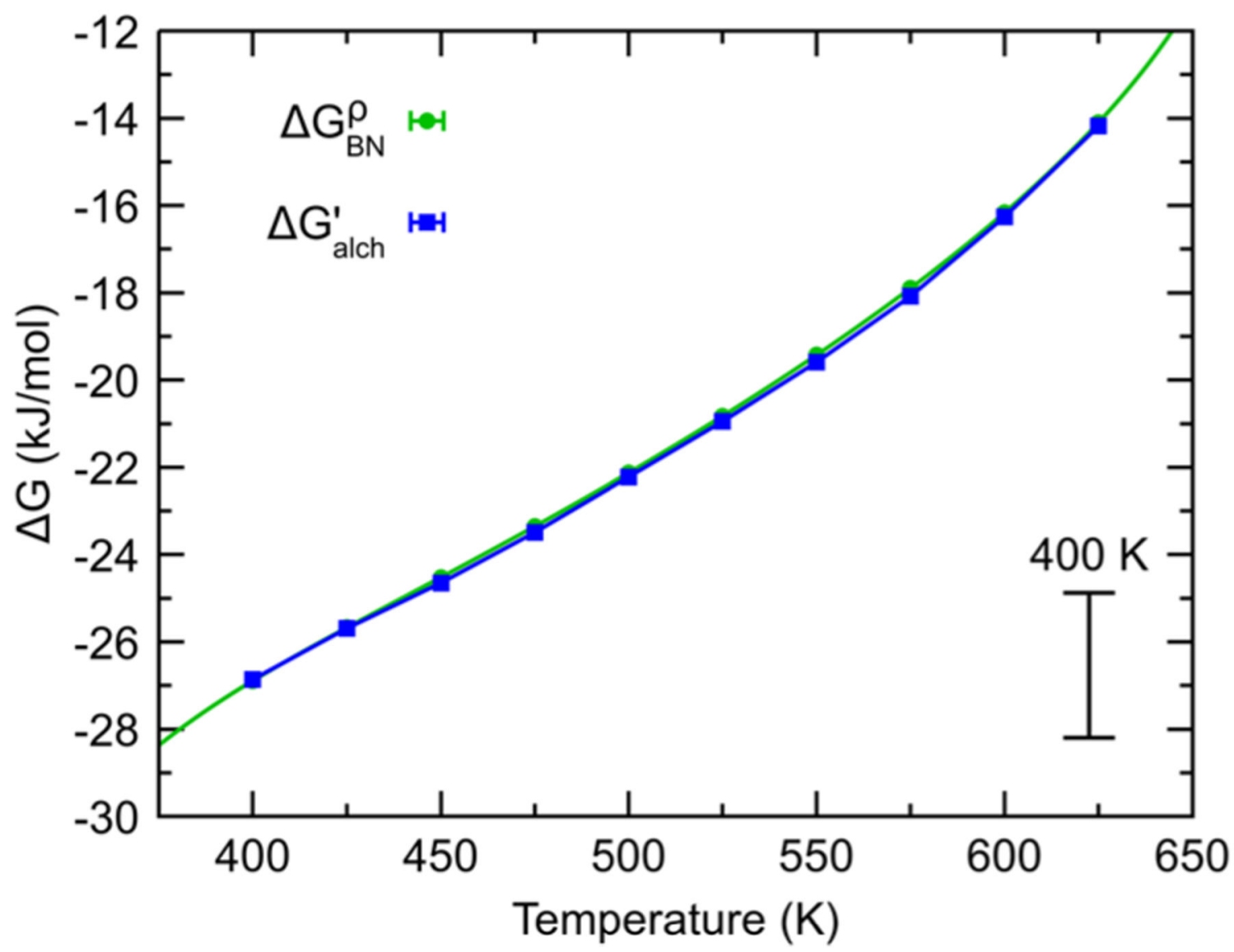

Figure 5.

Comparison of alchemical hydration free energy, $\Delta G_{\text {alch }}^{\prime}$, to the Ben-Naim density-based SFE, $\Delta G_{\mathrm{BN}}^{\rho}$. The scale bar indicates the size of the thermal energy, $R T$, at $400 \mathrm{~K}$. 


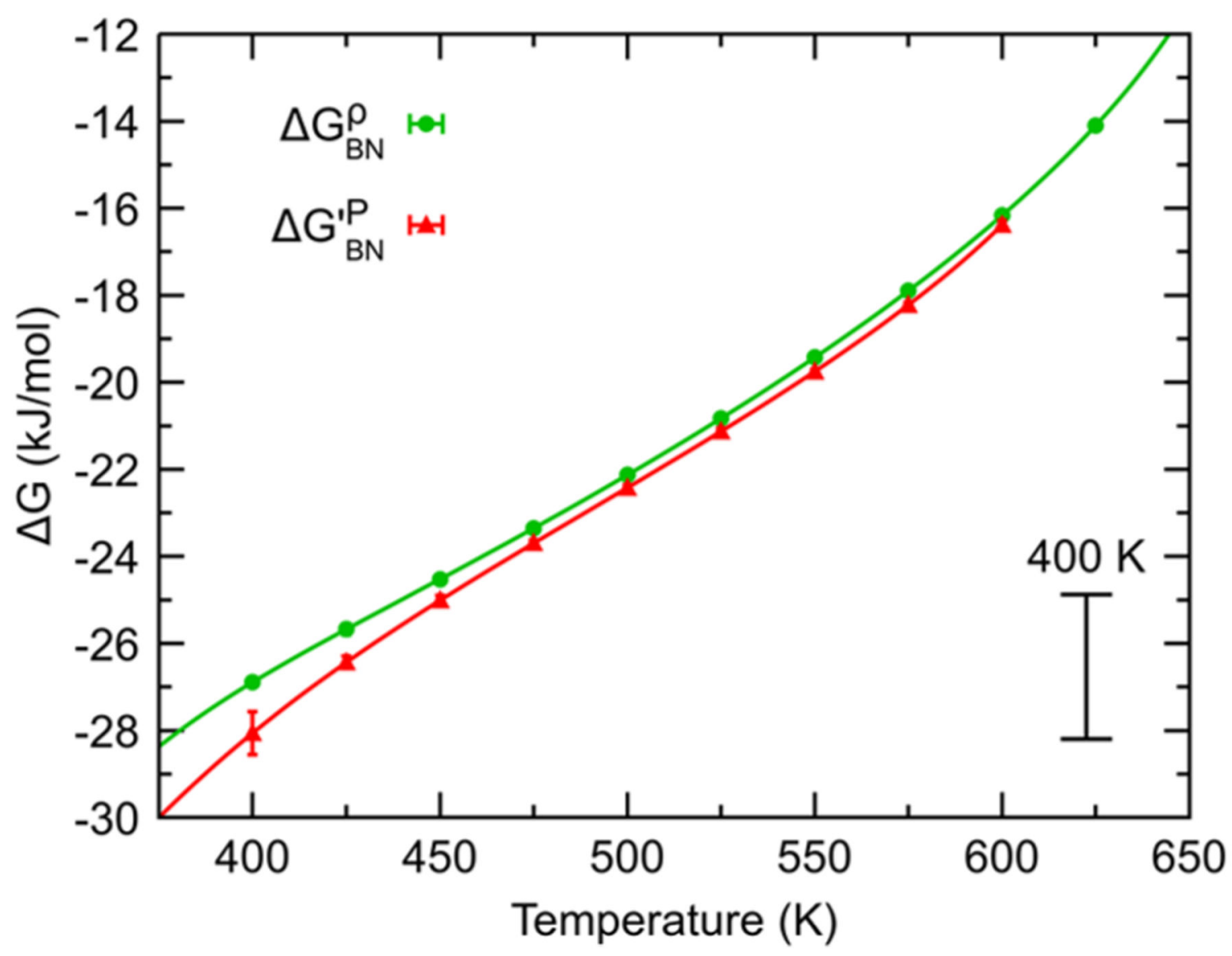

Figure 6.

Comparison of the density-based solvation free energy, $\Delta G_{\mathrm{BN}}^{\rho}$, and the virial expansion form of the pressure-based route, $\Delta G^{\prime}{ }_{\mathrm{BN}}^{P}$. The scale bar indicates the size of the thermal energy, $R T$, at $400 \mathrm{~K}$. 
Table 1.

Liquid and Gas Densities and Vapor Pressures Obtained from Slab Simulations ${ }^{a}$

\begin{tabular}{cccc}
\hline$T(\mathbf{K})$ & $\rho_{\mathbf{1}}(\mathbf{m o l} / \mathbf{L})$ & $\rho_{\mathrm{g}}(\mathbf{m o l} / \mathbf{L})$ & $\boldsymbol{P}_{\text {vap }}(\mathbf{b a r})$ \\
400 & $52.679 \pm 0.001$ & $0.0161 \pm 0.0002$ & $.38 \pm 0.06$ \\
425 & $51.635 \pm 0.001$ & $0.0362 \pm 0.0003$ & $1.01 \pm 0.05$ \\
450 & $50.470 \pm 0.002$ & $0.0715 \pm 0.0005$ & $2.25 \pm 0.07$ \\
475 & $49.180 \pm 0.003$ & $0.1328 \pm 0.0008$ & $4.49 \pm 0.09$ \\
500 & $47.748 \pm 0.005$ & $0.234 \pm 0.002$ & $8.30 \pm 0.2$ \\
525 & $46.176 \pm 0.006$ & $0.391 \pm 0.003$ & $14.00 \pm 0.2$ \\
550 & $44.396 \pm 0.006$ & $0.633 \pm 0.004$ & $22.60 \pm 0.2$ \\
575 & $42.386 \pm 0.008$ & $1.003 \pm 0.005$ & $34.60 \pm 0.3$ \\
600 & $40.051 \pm 0.008$ & $1.568 \pm 0.006$ & $52.10 \pm 0.1$ \\
625 & $37.200 \pm 0.010$ & $2.468 \pm 0.008$ & $75.40 \pm 0.2$ \\
\hline
\end{tabular}

${ }^{a}$ Uncertainties are the standard error of the mean. 
Table 2.

Comparison of Virial Coefficients of BLYPSP-4F Water and the Contributions of the Second and Third Terms in the Virial Equation of State (eq 10)

\begin{tabular}{ccccc}
\hline $\boldsymbol{T}(\mathbf{K})$ & $\boldsymbol{B}_{\mathbf{2}}(\mathbf{L} / \mathbf{m o l})$ & $\boldsymbol{B}_{\mathbf{3}}\left(\mathbf{L}^{2} / \mathbf{m o l}^{2}\right)$ & $\boldsymbol{B}_{2} \rho_{\mathrm{g}}$ & $\boldsymbol{B}_{\mathbf{3}} \rho_{\mathrm{g}}{ }^{2}$ \\
400 & -1.1862 & -2.8679 & -0.0191 & -0.0007 \\
425 & -0.8634 & -0.9642 & -0.0313 & -0.0013 \\
450 & -0.6541 & -0.3431 & -0.0468 & -0.0018 \\
475 & -0.5115 & -0.1209 & -0.0679 & -0.0021 \\
500 & -0.4105 & -0.0366 & -0.0960 & -0.0020 \\
525 & -0.3365 & -0.0039 & -0.1317 & -0.0006 \\
550 & -0.2806 & 0.0083 & -0.1776 & 0.0033 \\
575 & -0.2375 & 0.0123 & -0.2382 & 0.0124 \\
600 & -0.2034 & 0.0129 & -0.3190 & 0.0318 \\
625 & -0.1761 & 0.0121 & -0.4344 & 0.0740 \\
\hline
\end{tabular}




\section{올 \\ 일}

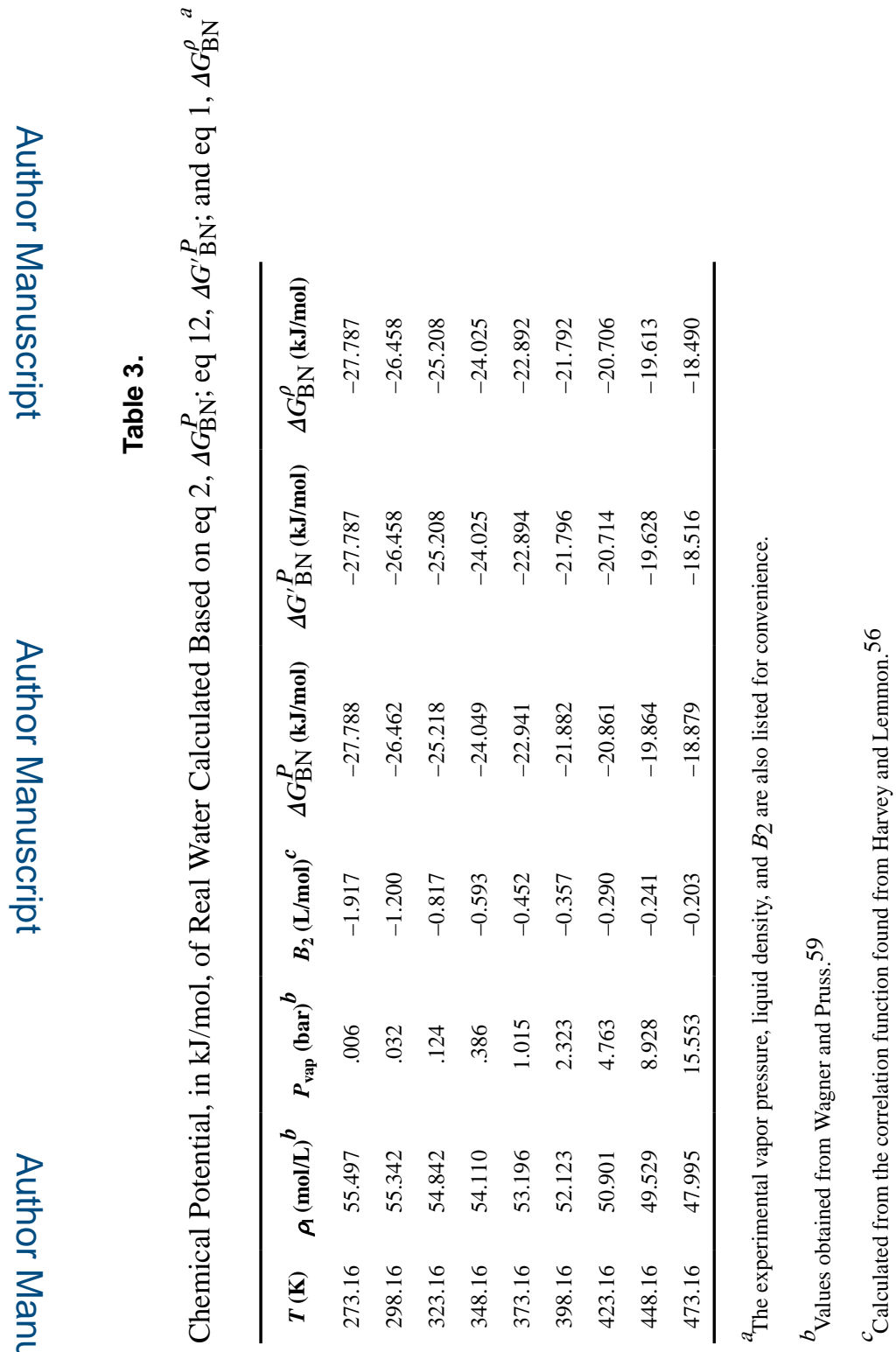

J Phys Chem B. Author manuscript; available in PMC 2021 February 06. 